\title{
Universidad y Medio Ambiente. Retos y desafíos hacia el desarrollo sostenible. Caso Universidad Metropolitana sede Machala
}

\section{University and Environment. Challenges and challenges towards sustainable development. Case of the Metropolitan University of Machala headquarters}

Sandy Jahaira Gonzaga-Añazco

Universidad Metropolitana, Machala, Ecuador

sgonzaga@umet.edu.ec

https://orcid.org/0000-0001-6214-6492

José Nicolás Molina-Sánchez

Universidad Metropolitana, Machala, Ecuador

jose.molina@est.umet.edu.ec

(D) https://orcid.org/0000-0002-8986-1434

María Paula Espinoza-Villacís

Universidad Metropolitana, Machala, Ecuador

maría.villacis.e@est.umet.edu.ec

https://orcid.org/0000-0003-0282-5375

Recepción: 11/08/2021 | Aceptación: 09/11/2021 | Publicación: 25/11/2021

Cómo citar (APA, séptima edición):

Gonzaga-Añazco, S.J., Molina-Sánchez, J.N., y Espinoza-Villacís, M.P. (2021). Universidad y Medio Ambiente. Retos y desafíos hacia el desarrollo sostenible. Caso Universidad

Metropolitana sede Machala. INNOVA Research Journal, 6(3.2), 14-31. https://doi.org/10.33890/innova.v6.n3.2.2021.1914

\section{Resumen \\ En los últimos años se ha evidenciado una latente necesidad profesional, dirigida a la inserción de los estudiantes universitarios en la solución de los problemas sociales que afectan a la comunidad. Por lo tanto, es indispensable diseñar actividades y acciones que posibiliten, desde la Universidad, el desarrollo de habilidades y capacidades para la conservación del Medio Ambiente. Es así como en este proceso se inserta la Universidad, en la que se desarrolla un proceso educativo destacando la importancia del conocimiento y cumplimiento de las normas ambientales. La Universidad Metropolitana del Ecuador se encuentra vinculada a la comunidad a través de diferentes proyectos}


y eventos académicos con enfoque de sostenibilidad, por lo tanto, con esta investigación se pretende conocer la percepción de la comunidad universitaria frente a las acciones ambientales generadas por la Universidad Metropolitana Sede Machala, involucrando en este estudio a los estudiantes, docentes y personal administrativo. La presente investigación es de tipo exploratoria, la metodología utilizada para el levantamiento de información tiene un enfoque cuantitativo, aplicando la técnica de encuestas. El objetivo de la investigación se centra en realizar un diagnóstico de la percepción medioambiental de la comunidad universitaria, para elaborar una línea base para futuras investigaciones encaminadas en plantear propuestas de mejora para el fortalecimiento de la cultura ambiental de la comunidad universitaria. Dentro de los principales hallazgos se encuentra el escaso conocimiento y desinterés del cuidado del medio ambiente, factores primordiales para la generación de cultura ambiental.

Palabras claves: universidad; medioambiente; desarrollo sostenible; responsabilidad social empresarial.

\begin{abstract}
In recent years, a latent professional need has been evidenced, aimed at the insertion of university students in the solution of social problems that affect the community. Therefore, it is essential to design activities and actions that allow, from the University, the development of skills and capacities for the conservation of the Environment. This is how the University is inserted in this process, in which an educational process is developed highlighting the importance of knowledge and compliance with environmental regulations. The Metropolitan University of Ecuador is linked to the community through different projects and academic events with a sustainability approach, therefore, this research aims to know the perception of the university community regarding the environmental actions generated by the Metropolitan University Machala headquarters, involving students, teachers and administrative staff in this study. This research is exploratory, the methodology used to collect information has a quantitative approach, applying the survey technique. The objective of the research focuses on making a diagnosis of the environmental perception of the university community, to develop a baseline for future research aimed at proposing improvement proposals to strengthen the environmental culture of the university community. Among the main findings is the scarce knowledge and lack of interest in caring for the environment, essential factors for the generation of environmental culture.
\end{abstract}

Keywords: university; environment; sustainable development; corporate social responsibility.

\title{
Introducción
}

La Universidad Metropolitana se encuentra vinculada a la comunidad a través de diferentes proyectos y eventos académicos con enfoque de sostenibilidad, lo cual motiva a la sociedad a implementar acciones proambientales. Por esta razón el desarrollo de la presente investigación busca sustentar la incógnita planteada: ¿Cómo percibe la Comunidad Universitaria las acciones ambientales en la UMET Sede Machala?, involucrando en este estudio a los estudiantes, docentes y personal administrativo.

Se han establecidos los siguientes objetivos específicos: Evaluar las acciones ambientales; determinar el conocimiento ambiental de la comunidad universitaria; establecer el nivel de compromiso de la comunidad universitaria para la implementación de acciones ambientales; 
identificar las acciones ambientales a implementar en la Universidad Metropolitana Sede Machala. Para lo cual se plantea una revisión del estado del arte de los principales ejes temáticos relacionados con los retos y desafíos que debe enfrentar la universidad como ente generador de conocimientos para contribuir a la creación de una cultura ambiental con enfoque de sostenibilidad.

\section{Medio Ambiente}

Bolaños Sánchez, Ortega Garnelo, y Baza Reyes (2015) señalan que en el mundo moderno el término medio ambiente adquiere valor debido a la creciente conciencia ambiental, tomando en consideración las complejas problemáticas que ocasionan efectos cada vez más irreversibles poniendo en riesgo los ecosistemas. Situaciones agravantes como el calentamiento global, las emisiones de dióxido de carbono, la fragilidad de ecosistemas terrestres y acuáticos, la extinción de múltiples especies y los distintos tipos de contaminación, son algunos de los factores que generan la expectativa de un futuro incierto para el planeta. Así mismo, el deterioro del medio ambiente a nivel general se evidencia a través del uso inconsciente de los recursos naturales, así como, la falta de atención o interés que los seres humanos otorgan a dicha problemática. Por otro lado, se han presentado ante la comunidad varios métodos, alternativas y actividades, las cuales se pueden implementar como acciones proambientales que contribuyan a marcar la diferencia en las situaciones presentes.

Para tener un panorama más extenso, Bybee (1991) indica que la sostenibilidad surge por vía negativa, como resultado de los análisis de la deteriorada situación ambiental. A su vez, Laportilla Estévez, et al. (2020) menciona que la sostenibilidad hace referencia a la atención de las necesidades actuales sin comprometer la capacidad de las generaciones futuras de poder satisfacer las suyas, garantizando un equilibrio ante el crecimiento económico, el cuidado del medio ambiente y el bienestar social, es lo que se conoce como Triple Vertiente de la Sostenibilidad. Por el contrario, Mckeown (2002) hace referencia a la sustentabilidad como un modelo para reflexionar en un futuro en donde se debe tomar en cuenta las consideraciones ambientales, económicas y sociales donde se valore la búsqueda de desarrollo hacia una mejor calidad de vida. Mientras que, Gallopín (2003) indica que el término de desarrollo sustentable es completamente diferente a la sustentabilidad, en la interpretación de que la palabra "desarrollo" se dirige a una noción de cambio, es decir un cambio que es paulatino y orientado. Lo que se sustenta y lo que se requiere ser sustentable es el mejoramiento de la condición y calidad humana. A lo que Morocho (2018) añade que una mentalidad consumista y un modelo de sistema de producción lineal ocasionarán en un futuro no muy lejano escases de recurso naturales, por lo que es necesario que a nivel mundial se implementen estrategias para lograr un desarrollo sostenible.

Así mismo, García (2018) menciona que el medio ambiente comprende varios factores relacionados con los recursos naturales, entre ellos tierra, agua, aire, flora y fauna; además de poder integrar recursos artificiales, como los bienes culturales. Por medio de esta definición planteada se puede asegurar que los seres humanos poseen la oportunidad y el derecho a vivir en un ambiente sano. Con respecto a esto la ONU (1987) manifiesta que el desarrollo sostenible es aquel que satisface las necesidades de la presente generación sin comprometer la capacidad de las generaciones futuras de satisfacer sus propias necesidades. Es decir, aprobar a que todos los pueblos del planeta puedan acceder $\mathbf{y}$ vivir en un nivel satisfactorio de desarrollo social, 
económico, de realización humana y cultural, en una tierra cuyos recursos serían empleados de manera consciente, preservando las especies y los medios. La ONU manifestó lo planteado en el informe Brundtland, en el cual se discutieron temas relevantes al medio ambiente, desarrollo sostenible, factores económicos, políticos y sociales que contribuyen a la mejora de un país como tal.

Vargas et al. (2017) consideran que la Educación Ambiental posee una base esencial para innovar la postura o la actitud de las relaciones del ser humano con su entorno para alcanzar una armonía entre ambos, generando un aumento en la calidad de vida y del ambiente, involucrando de modo conjunto las diversas áreas y su colaboración de la sociedad civil y las demás instituciones para otorgar mejores resultados para las generaciones futuras. Las instituciones educativas deben promover la generación de conciencia ambiental, por lo que es necesario implementar acciones dirigidas al cumplimiento de este objetivo. Por lo tanto, las instituciones deben entablar diversos acuerdos que contribuyan desde el factor de la educación, una mayor iniciativa hacia el cuidado y la preservación del medioambiente (Valera Mejía y Silva Naranjo, 2012).

Con respecto a la Responsabilidad Social Empresarial, Cancino y Morales (2008) señalan que son circunstancias donde las empresas u organizaciones se comprometen y cumple acciones que favorecen a la sociedad, enfocándose más allá de los intereses de la empresa y por sobre lo que se espera como cumplimiento de la ley. Aunque, para Sarmiento (2010) considera a la RSE como una filosofía o ideología corporativa adoptada por la alta dirección de la empresa para actuar en beneficio de sus propios trabajadores, sus familias y el entorno social en las zonas de influencia de las empresas. En el caso de Reyes de Blobfeld (2014) indica que se requiere invertir tiempo, talento y recursos por parte de cualquier empresa, para la construcción de un futuro con mayores oportunidades de crecimiento. Niño y Cortés (2018) menciona que la revolución digital demanda pensar la comunicación con enfoque multidimensional, puesto que debe estar orientada a satisfacer las necesidades y motivaciones de los diversos grupos de interés, a concentrarse en la razón de ser del negocio, a contribuir con acciones de alto impacto económico y saldo social, a generar contenidos y mensajes transparentes, auténticos y consistentes que generen confianza, credibilidad y reciprocidad.

En el año de 1997 se generó una conferencia para discutir, dar a conocer y proponer soluciones óptimas para el cambio climático, dicha reunión se determinó el Protocolo de Kioto desarrollado en Japón, el cual tiene como objetivos para los países involucrados reducir las emisiones de gases de efecto invernadero provocados por el medio ambiente al igual que el calentamiento global. Es indispensable conocer de dicho evento, ya que invita a los países industrializados a contribuir y reducir la contaminación atmosférica del planeta.

Por otro lado, surge la Agenda 2030 para el Desarrollo Sostenible, la cual tuvo aprobación en el año 2015 por parte de la Organización de las Naciones Unidas donde establece como principales objetivos mantener un equilibrio hacia la sostenibilidad política, económica, social y ambiental. Como lo plantea ONU (2019) la Agenda 2030 para el Desarrollo Sostenible, que incluye 17 Objetivos y 169 metas, presenta una visión ambiciosa y completa del desarrollo sostenible en los factores económicos, sociales y ambientales.

Los Objetivos de Desarrollo Sostenible o también conocidos como ODS son 17 considerados de carácter global, cabe recalcar que fueron formulados de manera precisa y clara 
para que los Estados, organizaciones e individuos puedan realizar los cambios necesarios para alcanzar cada uno de los objetivos planteados. Aunque es un camino largo de recorrer, se requiere de compromiso y fidelidad. CEPAL (2016) presenta los 17 objetivos de desarrollo sostenible, mismos que hacen referencia a indicadores involucrados con el medio ambiente como el objetivo 6: garantizar la disponibilidad de agua y su gestión sostenible y el saneamiento para todo; el objetivo 12: garantizar las modalidades de consumo y producción sostenible; el objetivo 13: adoptar medidas urgentes para combatir el cambio climático y sus efectos; el objetivo 15: promover el uso sostenible de los ecosistemas terrestres, luchar contra la desertificación, detener e invertir la degradación de las tierras y frenar la pérdida de la diversidad biológica. Al respecto, Gómez Gil (2018) sostiene que los ODS poseen una versión distinta a los ODM haciendo referencia a los Objetivos de Desarrollo del Milenio, a su vez los ODS incorporan factores más cruciales que vinculan aspectos económicos, sociales y ambientales.

\section{Universidad}

Según Aparicio Macanchi y Toledo (2017) la universidad como ente social posee un rol importante para la sociedad, establece interrelaciones de manera activa en el desarrollo económico, social y cultural. Para dar respuesta a este compromiso social, se aboca a involucrarse en la preservación, desarrollo y promoción de la cultura, concebidas como funciones que se concretan en la ejecución de los procesos universitarios: docencia, investigación y vinculación.

Toda institución sea pública o privada, posee un compromiso con la sociedad y el medio ambiente, en el cual desarrolla sus actividades económicas, con el propósito de minimizar su impacto negativo por el consumo de energías no renovables, por lo tanto, es indispensable implementar la Responsabilidad Social Empresarial en cada una de las funciones sustantivas que involucra a la educación, estableciendo las pautas necesarias para reducir su contaminación en el territorio donde funciona la entidad.

En lo concerniente a los aspectos legales, que regulan el funcionamiento de las instituciones de educación superior, se encuentran: el Consejo de Aseguramiento de la Calidad de la Educación Superior CACES y la Secretaría de Educación Superior, Ciencia, Tecnología e Innovación SENESCYT. El CACES centra sus funciones en regulación, coordinación y planificación los procesos participativos de acompañamiento, evaluación, acreditación y cualificación para garantizar el desarrollo de una cultura de la calidad en las instituciones de educación superior, enfocada en el equilibrio de la docencia, la investigación e innovación y la vinculación con la sociedad. Esta institución se ha encargado de realizar procesos de evaluación, acreditación y aseguramiento de la calidad en el área de la educación desde el 2011; su finalidad, es mostrar un nuevo enfoque sobre políticas de evaluación institucional de entidades de educación superior, justamente para asegurar la calidad y que ésta responda a las necesidades y requerimientos actuales del país y a nivel internacional, la misma que se encuentra en la Ley Orgánica de Educación Superior LOES.

La Secretaría de Educación Superior, Ciencia, Tecnología e Innovación se enfoca en ejercer la rectoría de la política pública de educación superior, ciencia, tecnología y saberes ancestrales y gestionar su aplicación, con enfoque en el desarrollo estratégico del país. Coordinar las acciones entre el Ejecutivo y las instituciones de educación superior en aras del fortalecimiento 
académico, productivo y social. SENESCYT, es una institución pública encargada de velar por el cumplimiento de la política pública, en donde su misión es coordinar acciones de la Función Ejecutiva con las instituciones de educación superior. Además, en lo que respecta a la educación superior, se busca garantizar el acceso gratuito, ofertar carreras y programas de formación académica que impulsen la dinamización de la economía que están alineadas con las políticas de gobierno a mediano plazo.

La Universidad Metropolitana del Ecuador (UMET), es una entidad de educación superior que fue creada por Ley 2000-14 por el Congreso Nacional del Ecuador, la misma que fue publicada en el Registro Oficial 68, el 2 de mayo del 2000. La matriz de esta institución educativa se encuentra localizada en la ciudad de Guayaquil y a su vez posee sedes en las ciudades de Quito y Machala. En lo concerniente a la UMET Sede Machala, esta se encuentra actualmente conformada por 30 colaboradores como personal administrativo y 53 docentes.

En el aspecto legal, la Universidad Metropolitana del Ecuador se conformó como persona jurídica con autonomía tanto académica como a nivel de gestión administrativa y financiera; es una institución sin fines de lucro, con arraigado enfoque de inclusión que respeta todas las formas de pensamiento universal a nivel científico.

En este ámbito, la Universidad Metropolitana del Ecuador, posee un fuerte compromiso con el medioambiente, no solo en el uso de energías no renovables para efectuar sus actividades de prestación de servicios de formación profesional, sino también considera que es su responsabilidad capacitar y fomentar la educación hacia la protección del medio ambiente, tanto a la comunidad universidad como a la población en la cual desarrolla sus actividades, con la finalidad de mejorar la calidad de vida, salvaguardando la flora y fauna del entorno.

\section{Metodología}

Se ha identificado que la presente investigación tiene un alcance exploratorio, ya que según Hernández Sampieri y Mendoza Torres (2018) los estudios exploratorios se llevan a cabo cuando el propósito es examinar un fenómeno o problema de investigación nuevo o poco estudiado, sobre el cual se tienen muchas dudas o no se ha abordado antes. Los estudios exploratorios también sirven para obtener información sobre la posibilidad de llevar a cabo una investigación más completa y profunda respecto de un contexto particular, identificar conceptos o variables promisorias a indagar, establecer prioridades para futuros estudios o sugerir afirmaciones, hipótesis y postulados. Tal como el caso de la identificación de la percepción de la comunidad universitaria frente a las acciones proambientales implementadas por la Universidad Metropolitana sede Machala, con la finalidad de generar investigaciones posteriores que proporcionen hallazgos significativos que puedan ser aplicados para potencializar aquellas acciones que han tenido un mayor impacto en la comunidad universitaria e implementar acciones de mejora en aquellos aspectos percibidos como debilidades.

La metodología utilizada para el levantamiento de información tiene un enfoque cuantitativo, mismo que es el apropiado cuando se pretende estimar las magnitudes u ocurrencia de los fenómenos con la finalidad de probar hipótesis. El diseño de la investigación fue de carácter documental y de campo, considerando que Posada González (2017) señala a la investigación 
documental como un proceso basado en la búsqueda, recuperación, análisis, crítica e interpretación de datos secundarios, es decir, los obtenidos y registrados por otros investigadores en fuentes documentales: impresas, audiovisuales o electrónicas, mientras que la investigación de campo consiste en la recolección de datos directamente de los sujetos investigados, o de la realidad donde ocurren los hechos, es decir la obtención de datos de fuentes primarias, sin manipular o controlar variable alguna. Para lo cual se utilizó un muestreo aleatorio simple con la información de la población a investigar 888 personas, clasificadas en: 800 estudiantes, 58 docentes y 30 administrativos; se utilizó un nivel de confianza del $90 \%$ y un margen de error de 5\%, obteniendo una muestra de 208 personas, en las cuales se aplicó la técnica de encuestas, por medio de la generación de un cuestionario.

\section{Resultados}

La Universidad Metropolitana comprometida no solo con la educación de calidad, sino también en cumplimiento de su Responsabilidad Social Empresarial, ha promovido diversas acciones de formación en protección del medio ambiente, con la finalidad de fomentar una cultura verde en la comunidad y en la sociedad en general, para mejorar la calidad de vida de todos los seres vivos y de esta manera lograr disminuir el impacto negativo de la contaminación por las actividades industriales y del hogar que causan graves daños al planeta.

Consecuentemente, dentro de las acciones y responsabilidades que poseen las instituciones de educación superior, existe una actividad denominada Vinculación con la Sociedad, en la cual participan tanto docentes como estudiantes en el diseño programas y proyectos que coadyuven al mejoramiento del entorno, creando conciencia ambiental en la población. De esta forma, se cumplen dos objetivos: permitir a los estudiantes poner en práctica los conocimientos adquiridos en aulas y compartirlos con la comunidad, a su vez la ciudadanía adquiere nuevos conocimientos que les permitan mejorar su calidad de vida y su desarrollo económico, lo cual impacta a nivel general.

En cuanto a las acciones ambientales que ha ejecutado hasta el momento la Universidad Metropolitana sede Machala tanto a nivel interno como externo son las siguientes:

- Crear conciencia sobre el uso de energía eléctrica, agua, impresiones de hojas, entre otros.

- Reciclaje dentro de las instalaciones.

- Separación de materiales orgánicos de otro tipo de materiales.

- Propuesta de creación de objetos a partir de materiales reciclados.

- Educación ambiental dentro de la UMET y posteriormente esta actividad la comparten los estudiantes con la comunidad.

- Crear y mantener espacios verdes dentro y fuera de las instalaciones.

- Participar de actividades externas en donde pedían voluntarios para hacer limpieza de entornos naturales.

- Visitas posteriores de control para corroborar el impacto de la educación ambiental implementada en sectores que recibieron el apoyo y formación por parte de la UMET. Mencionando a la encuesta realizada a la comunidad universitaria, de las diez preguntas planteadas en la encuesta se seleccionó las de mayor relevancia y las que más impacto han creado 
con respecto a la problemática que se plantea. Cabe mencionar, que al haber ejecutado la encuesta se empleó el muestreo aleatorio simple para determinar el tamaño de la muestra de 208 personas a encuestar.

Una de las preguntas con mayor relevancia en la investigación hace referencia a la percepción que posee la comunidad universitaria con respecto a la gestión ambiental de la UMET. Señalando lo mencionado por Ghitis y Alba (2019) las percepciones son únicas y a su vez se apoyan bajo los entendimientos y las costumbres previas. Poseen tres particularidades, las cuales son subjetivas, selectivas y temporales. Las subjetivas dando a entender que se acate bajo las personas, así mismo las selectivas bajo las señales trasmitidas y receptadas por nuestro cerebro y, por último, los temporales indicando que la percepción de cada individuo puede cambiar a partir de nuevas experiencias y conocimientos que se trasmiten y se generan día a día.

La participación comunitaria es un importante instrumento para transitar hacia este tipo de desarrollo a escala territorial, en apoyo a las instituciones responsabilizadas en la protección del medio ambiente y un apoyo primordial lo constituyen las escuelas en su función de integrar la dimensión ambiental en el sistema educativo dirigido a la adquisición de conocimientos, habilidades, y la formación de valores éticos. Según la investigación de Cuéllar y Méndez (2006)

\section{Gráfico 1}

\section{Percepción de la gestión ambiental}

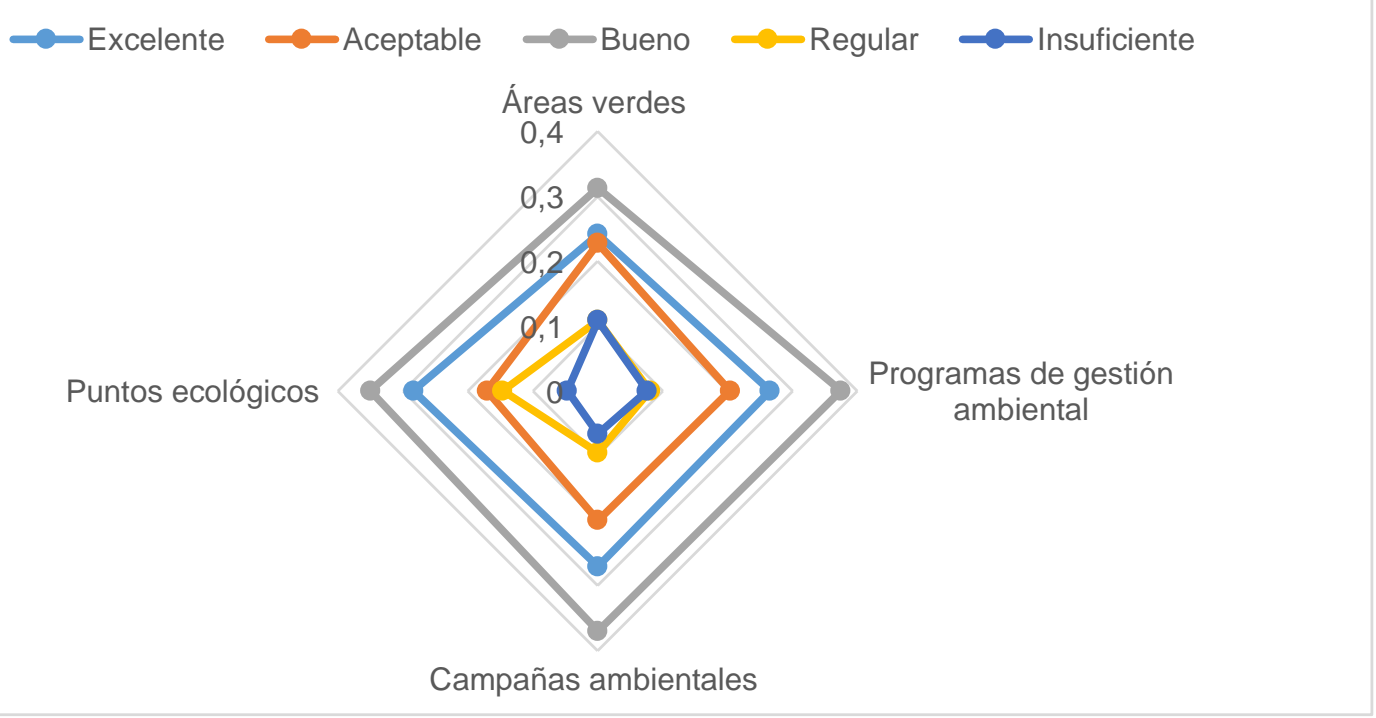

Fuente: Elaboración propia

Con esta pregunta se pretende conocer la percepción de los miembros de la comunidad universitaria frente a acciones específicas implementadas por la UMET sede Machala en beneficio del medioambiente. La manera en la que se encuentra estructurada dicha pregunta es a base de una escala de Likert la cual se dirige desde Excelente hasta Insuficiente, así brinda un mayor alcance para visualizar los resultados obtenidos. En el gráfico radial se visualizan cuatro apartados: áreas verdes, programas de gestión ambiental, campañas medioambientales y puntos ecológicos; donde 
se puede evidenciar que el aspecto con mejor percepción son los puntos ecológicos, mientras que el área con peor percepción para la comunidad universitaria son las áreas verdes, factor relevante a considerar para la implementación de futuras acciones.

El 24,04\% de los encuestados perciben las acciones proambientales implementadas por la Universidad Metropolitana sede Machala como "excelente", mientras que el 31,25\% de la comunidad resalta que la gestión ambiental es adecuada. Así mismo, el 23,08\% señala que esta es aceptable, el 11,06\% regular y el 11,06\% insuficiente. Por lo tanto, se puede apreciar que, aunque la mayoría de los encuestados tiene una percepción positiva, aún existe un gran porcentaje que no están conformes con dichas acciones, lo cual constituye una oportunidad de mejora.

Como parte de los objetivos de la investigación se encuentra conocer el nivel de aceptación de la comunidad universitaria frente a las diversas acciones ambientales que ha implementado la Universidad Metropolitana, tales como el Congreso Internacional de Medio Ambiente y Desarrollo Sostenible efectuado desde el año 2016 donde se presentan conferencistas de renombre con la intención de propiciar una cultura ambiental ante los participantes, mediante temáticas de distinta tendencia, innovación y buenas prácticas. Uno de los principales objetivos que brinda el Congreso Internacional de Medio Ambiente y Desarrollo Sostenible es fomentar el avance académico y científico por medio del cambio y expansión de las actividades relacionadas con la enseñanza, la exploración y la vinculación con la sociedad en las diversas áreas del conocimiento (UMET, 2019). En la siguiente gráfica se presentan otros factores como el tiempo de ejecución de las acciones, las capacitaciones brindadas a la comunidad universitaria, la percepción del medio ambiente como una moda y no como un factor de constante importancia, y la relación entre el consumo y medioambiente.

\section{Gráfico 2}

\section{Percepción de acciones ambientales}

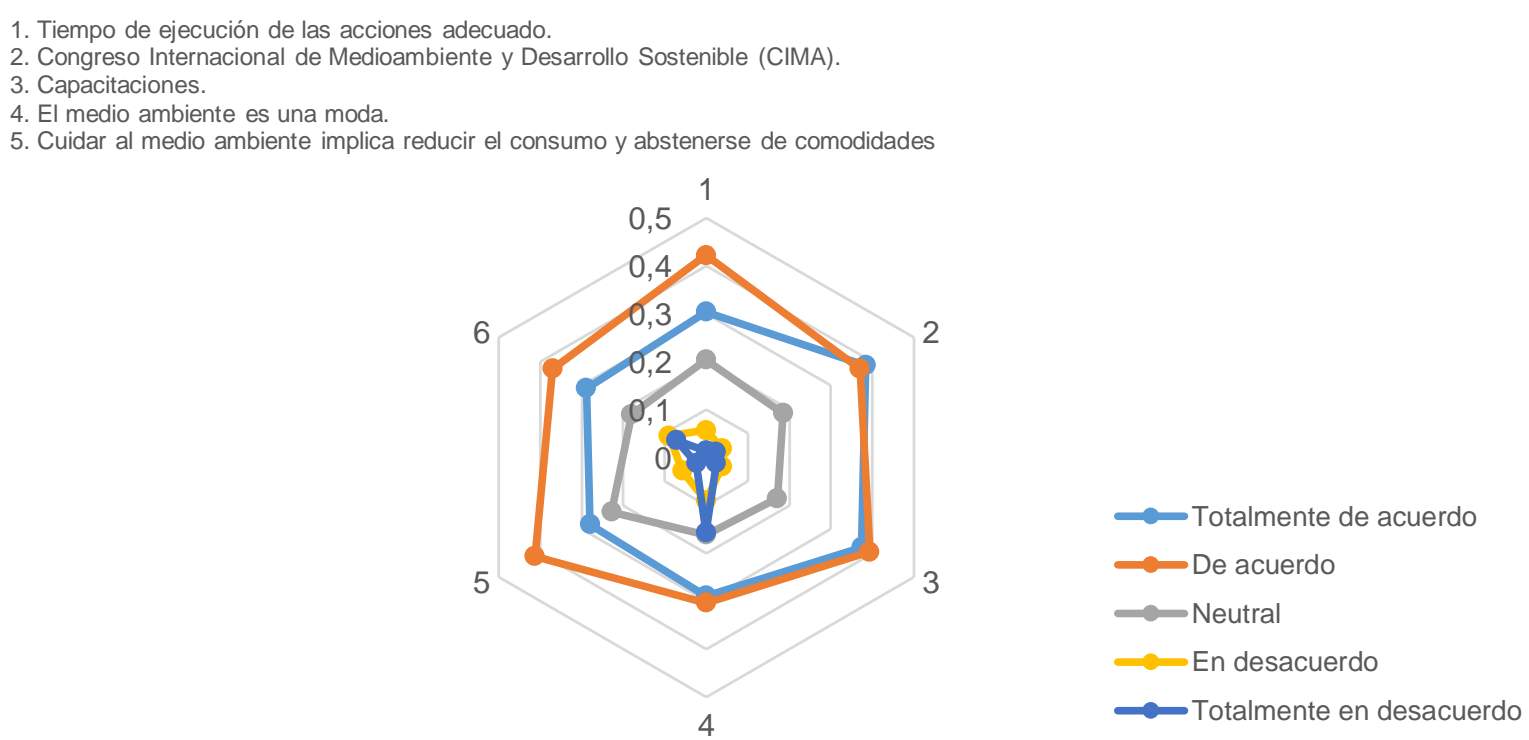

Fuente: Elaboración propia 
La pregunta anterior hace referencia a la percepción de la comunidad universitaria respecto a las acciones ambientales implementadas por la Universidad Metropolitana sede Machala en beneficio del medio ambiente. Los ítems evaluados son: el tiempo de ejecución de las acciones, los Congresos Internacional del Medio Ambiente y Desarrollo Sostenible, al igual que, las capacitaciones establecidas hacia la comunidad universitaria, donde el $42,31 \%$ de los encuestados están de acuerdo, el 30,29\% con totalmente de acuerdo, 20,19\% tiene una posición neutral, el $5,77 \%$ en desacuerdo y el $1,44 \%$ totalmente en desacuerdo con las acciones aplicadas.

De acuerdo con Pardavé (2007) el reciclaje se cimienta en, que los residuos deben ser tratados como recurso, para luego, reducir la demanda de recursos naturales y la cantidad de materia que requieran una disposición final. Según Jurado (2021) el reciclaje como la operación compleja que permite la recuperación, transformación y elaboración de un material a partir de residuos, ya sea total o parcial en la composición definitiva.

\section{Gráfico 3}

Disposición a proteger y conservar el medioambiente

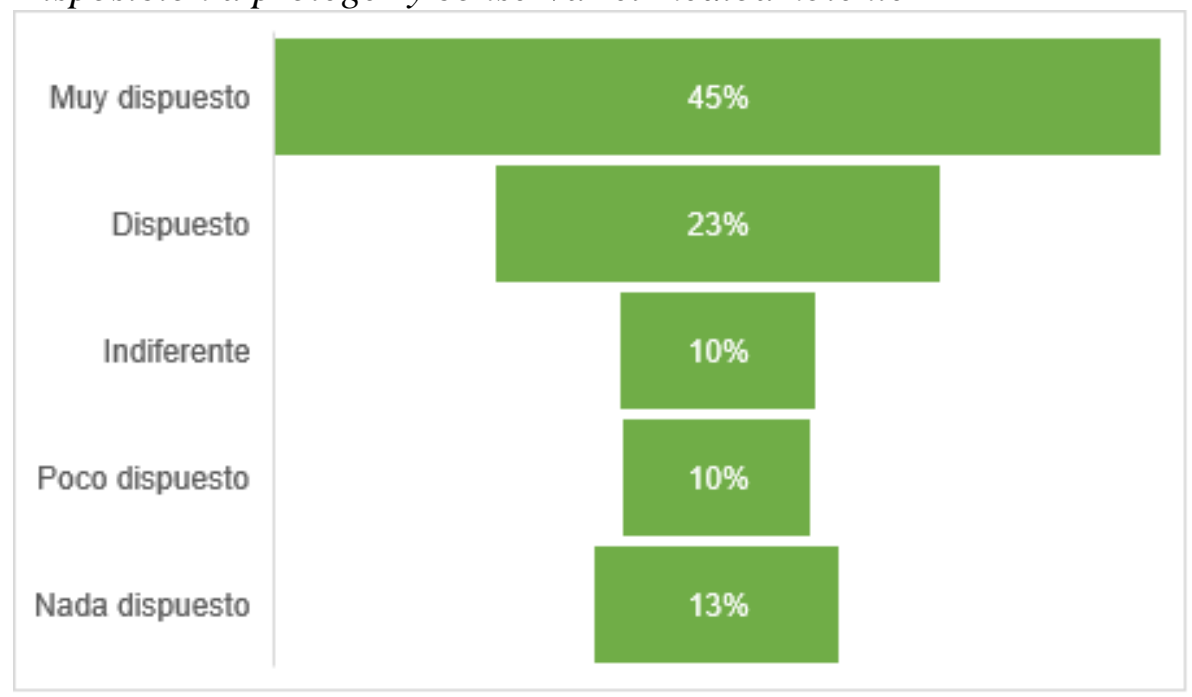

Fuente: Elaboración propia

En el gráfico anterior se pretende conocer la disposición de la comunidad universitaria a la protección y conservación del medioambiente, que no solo es trabajo de las instituciones educativas, sino de toda su comunidad, la cual debe adquirir conocimientos y habilidades que le permitan el buen uso de recursos y a su vez contribuir a la comunidad en general. Los principios de sustentabilidad se encuentran declarados en la Misión de la Universidad Metropolitana, siendo uno de los factores primordiales la generación de una cultura ambiental orientada al cumplimiento de los valores institucionales. En base a lo antes mencionado es relevante conocer qué tan dispuesta se encuentra la comunidad universitaria a implementar y promover acciones para proteger y conservar el medio ambiente, cuyos resultados se presentan en el siguiente gráfico.

Con relación al gráfico de que tan dispuestos se encuentran los estudiantes, se demuestra que el $45,2 \%$ indica estar "muy dispuestos" a colaborar con proyectos, actividades, campañas, 
entre otros aspectos que permitan realizar procesos de reciclaje dentro de su alma mater, con la finalidad de aportar su ayuda frente a esta problemática mundial que repercute en cada uno de los individuos y sus estilos de vida. De igual forma los estudiantes universitarios se encuentran en el interés de lograr la protección y conservación ambiental por medio de las distintas actividades de reciclaje.

\section{Análisis de problemas}

Surge la necesidad de incluir el análisis de problemas o también conocido como árbol de problemas que ejerce un papel fundamental en el desarrollo del documento, otorgando una visión más amplia sobre la relación de la universidad y el medio ambiente por medio del análisis estructurado. Como lo manifiesta Hernández-Hernández y Garnica-González (2015) el análisis de problemas tiene como fin poder desarrollar e interpretar ideas ingeniosas para reconocer las potenciales causas del problema, produciendo de una manera adecuada un modelo que exprese las razones y las consecuencias del problema. Por otro lado, Escobar y Escobar (2018) manifiesta que el árbol de problemas brinda al equipo o grupo de trabajo poder determinar la problemática y aplicar las estrategias. Además, de que facilita el orden de las ideas y con su objetividad se pretende encontrar el problema central. Mientras que, Maldonado-Villalpando et al. (2018) además de realizar el análisis del problema para un proyecto e identificar los factores negativos de una situación, presenta las variables causa-efecto entre los problemas analizados.

El principal problema percibido en la presente investigación es la escasa consciencia y educación ambiental por parte de la comunidad universitaria. A partir, del problema central o tronco haciendo referencia al árbol, se desglosan por medio de las ramas las causas o variables independientes y, por otro lado, a las variables dependientes o los efectos. Las cruciales causas expuestas son la falta de información ambiental, la falta de interés, la falta de cultura y la producción y acumulación de basura. Para obtener una orientación más amplia del problema central y las causas principales, se desprende las causas secundarias. En el caso de la falta de información ambiental se dirige a que la universidad no se enfoca en entablar información clara y precisa. En la falta de interés se clasifica en dos apartados el primero que no tienen tiempo y piensan que los problemas ambientales son pasajeros. La falta de cultura relacionada a que presentan escasos valores para resolver problemas ambientales y por último la causa de la producción y acumulación de basura dirigida a que poseen un desconocimiento en los procesos de reciclaje.

Luego de las causas desarrolladas, se muestran los efectos surgidos del problema central los cuales son la afectación de la imagen de la institución educativa, el uso inconsciente de los recursos, el no poseer un pensamiento y una ética ecológica orientada sobre los efectos que genere los efectos del medio ambiente y el deterioro ambiental son los efectos directos inmediatos del problema. Así mismo, de cada uno de los efectos se separa los efectos secundarios, en la afectación de la imagen de la institución educativa se relaciona con el bajo ingreso de estudiantes en los próximos semestres, el uso inconsciente de los recursos trae consigo el agotamiento de los recursos naturales, el no poseer un pensamiento y una ética ecológica orientada sobre los efectos que genere los efectos del medio ambiente se encuentra con el efecto de llegar a tener un riesgo ambiental y como último, determinando el deterioro ambiental repercute al calentamiento global. 


\section{Análisis de involucrados}

Según Maldonado Pérez, Pérez de Maldonado, y Bustamante Uzcátegui (2007) El análisis de los involucrados permite detectar intereses, problemas percibidos, recursos y mandatos, intereses en una estrategia y conflictos potenciales. A partir de esta información el interés se centra en la determinación del problema focal o central y sus causas.

Se debe identificar todos los grupos posibles que puedan relacionarse con la investigación, una vez identificados los grupos se debe realizar un análisis minucioso de cada uno de ellos en el que debe constar los intereses y necesidades: los posibles problemas a los que se tendrán que enfrentar dentro de los grupos de trabajo. Como primer punto, se definieron 7 grupos los cuales fueron; estudiantes, docentes, personal administrativo, personal de limpieza y seguridad, consejo estudiantil y consejo universitario.

El análisis de involucrados -grupos de interés, "stakeholders" o simplemente partes interesadas en el proyecto, es esencial en el Marco Lógico, por cuanto el éxito de un proyecto depende, en gran medida, del grado de contribución de algunos grupos a la intervención y de la oposición de otros, que no están de acuerdo en darle solución al problema. Por estas razones, el análisis inicial se revisará periódicamente, para incorporar los cambios de opinión de algún grupo sobre de sus intereses.

En el análisis de involucrados, interesa conocer los grupos interesados o afectados, de una $\mathrm{u}$ otra forma, por el proyecto, sean beneficiarios potenciales, personas que ven en peligro sus intereses; instituciones públicas y privadas que financian proyectos comunitarios, entre otros. Además, es indispensable conocer los intereses de cada grupo en relación con la solución del problema planteado. A este respecto, habría que distinguir entre los intereses de quienes apoyan una determinada estrategia para llevar a cabo el proyecto, y los de aquellos que se opondrían a esa estrategia, convirtiéndose en socios conflictivos, por considerar al proyecto una amenaza a sus objetivos particulares. Otro elemento que se debe considerar es el poder, mandato legal o estatutario, entendido como la capacidad de incidir directa o indirectamente sobre una determinada propuesta. Cada grupo tiene poder para apoyar u obstaculizar el desarrollo del proyecto, para buscar las estrategias idóneas para maximizar el apoyo y minimizar la resistencia de los grupos, cuando el proyecto se comience a ejecutar.

\section{Tabla 1}

Matriz de análisis de involucrados

\begin{tabular}{llll}
\hline \multicolumn{1}{c}{ Grupo } & \multicolumn{1}{c}{ Intereses } & Problemas percibidos & Recursos y mandatos \\
\hline \multirow{2}{*}{ Estudiantes } & $\begin{array}{l}\text { Tener conciencia acerca } \\
\text { de los problemas } \\
\text { ambientales. }\end{array}$ & Falta de cultura ambiental. & $\begin{array}{l}\text { R: Generar una imagen de } \\
\text { cuidado ambiental por parte de } \\
\text { los estudiantes de la Universidad } \\
\text { Poco conocimiento acerca de } \\
\text { cómo clasificar los residuos. }\end{array}$ \\
$\begin{array}{l}\text { Incentivar a los } \\
\text { estudiantes acerca el } \\
\text { cuidado ambiental. }\end{array}$ & $\begin{array}{l}\text { Falta de recursos por parte } \\
\text { del Consejo Universitario }\end{array}$ & $\begin{array}{l}\text { R: Desarrollar un plan } \\
\text { presupuestario para realizar } \\
\text { actividades de interés (medio }\end{array}$
\end{tabular}




\begin{tabular}{|c|c|c|c|}
\hline Grupo & Intereses & Problemas percibidos & Recursos y mandatos \\
\hline & & $\begin{array}{l}\text { para la implementación } \\
\text { actividades. }\end{array}$ & $\begin{array}{l}\text { ambiente). M: Representar los } \\
\text { intereses de la comunidad } \\
\text { universitaria en cuanto al medio } \\
\text { ambiente. }\end{array}$ \\
\hline $\begin{array}{l}\text { Personal } \\
\text { Administrativo }\end{array}$ & $\begin{array}{l}\text { Mejor manejo de los } \\
\text { recursos de su entorno. } \\
\text { Mejor la organización de } \\
\text { reciclaje y cuidado del } \\
\text { medio ambiente. } \\
\text { Mejorar la organización }\end{array}$ & $\begin{array}{l}\text { Deficiencia al momento de } \\
\text { desechar los residuos } \\
\text { (papeles, cartuchos de tinta, } \\
\text { bolsas plásticas). }\end{array}$ & $\begin{array}{l}\mathrm{R} \text { : Aplicación de capacitaciones } \\
\text { relacionadas a la temática de } \\
\text { reciclaje. }\end{array}$ \\
\hline $\begin{array}{l}\text { Personal de } \\
\text { Limpieza y } \\
\text { Seguridad }\end{array}$ & $\begin{array}{l}\text { de reciclaje y cuidado del } \\
\text { medio ambiente } \\
\text { Mejor manejo de los } \\
\text { recursos de su entorno. }\end{array}$ & $\begin{array}{l}\text { Desconocimiento sobre el } \\
\text { buen manejo de los desechos } \\
\text { dentro de la institución. }\end{array}$ & $\begin{array}{l}\text { R: Impartir capacitaciones para } \\
\text { disminuir la huella ambiental. }\end{array}$ \\
\hline $\begin{array}{l}\text { Consejo } \\
\text { Estudiantil }\end{array}$ & $\begin{array}{l}\text { Implementar actividades } \\
\text { las cuales ayuden a tomar } \\
\text { conciencia del cuidado } \\
\text { ambiental. }\end{array}$ & $\begin{array}{l}\text { Falta de actividades por parte } \\
\text { del Consejo Estudiantil. } \\
\text { Leve iniciativa hacia el } \\
\text { cuidado del medio ambiente. }\end{array}$ & $\begin{array}{l}\text { R: Desarrollo de un manual de } \\
\text { cuidado del medio ambiente } \\
\text { dentro de la Universidad } \\
\text { Metropolitana dirigido a } \\
\text { estudiantes y docentes. }\end{array}$ \\
\hline $\begin{array}{l}\text { Consejo } \\
\text { Universitario }\end{array}$ & $\begin{array}{l}\text { Ejecutar un plan de } \\
\text { mejora con relación a } \\
\text { políticas y actividades } \\
\text { ambientales. }\end{array}$ & $\begin{array}{l}\text { Falta en el renombre de las } \\
\text { leyes planteadas para el } \\
\text { cuidado del medio ambiente } \\
\text { y el entorno universitario. }\end{array}$ & $\begin{array}{l}\text { M: Optar por la creación de una } \\
\text { alianza con una asociación } \\
\text { vinculada al cuidado ambiental. }\end{array}$ \\
\hline
\end{tabular}

Fuente: Elaboración propia

La estructura de la matriz de análisis de involucrados se encuentra constituida de la siguiente manera: En la primera columna se incluyen los grupos involucrados directa o indirectamente en el proyecto, sin considerar personas. En la segunda columna se describen, los intereses de cada grupo, en relación con el problema seleccionado como problema central. Los intereses también pueden reflejar soluciones sugeridas por cada grupo que esté perjudicado por el problema. En la tercera columna se describen los problemas específicos o condiciones negativas, tal como son percibidos por cada grupo de involucrados, en relación con el problema en estudio. En la cuarta columna se hace referencia a los recursos y mandatos de los grupos; los recursos son los medios financieros y no financieros que los grupos pueden poner a disposición del proyecto, para contribuir a solucionar el problema (o para bloquear una solución propuesta); los mandatos vienen dados por la autoridad formal de cada grupo para proporcionar un servicio o cumplir una función determinada, que contribuya al éxito del proyecto.

Al final del análisis, lo ideal es lograr una mayoría a favor del proyecto, producto de la consecución de acuerdos y la satisfacción de los intereses de las partes involucradas. De esta forma, se evitan o minimizan los conflictos, y sería menor el impacto negativo en el proyecto. Este análisis se hace para identificar y esclarecer qué grupos y organizaciones están directa o indirectamente involucrados en el problema de desarrollo específico que intentamos resolver, para tomar en consideración sus intereses, su potencial y sus limitaciones. 
Una vez estructurados los grupos de la matriz del análisis de involucrados, se procede a la estructuración y búsqueda de los intereses de la comunidad universitaria. Por parte del primer grupo y el más significativo dentro de la investigación, los intereses de los estudiantes se basan en obtener conciencia acerca de los problemas ambientales, haciendo referencia a que se encuentra completamente interesados en los distintos aspectos del cuidado del medio ambiente, por otro lado, el grupo de personal administrativo tiene como interés el mejor manejo de los recursos y de igual forma mejorar la organización del reciclaje dentro de la unidad educativa. Con relación al grupo de personal de limpieza y seguridad, se puede relacionar que sus intereses van desde mejorar el reciclaje hasta el mejor manejo de los recursos, sin duda alguna un grupo de suma importancia, al momento de reciclar y capacitar acerca de los problemas ambientales. Otro grupo a resaltar es el consejo estudiantil, el cual su principal interés es el de implementar diversas actividades las cuales logren generar grandes impactos dentro de los jóvenes universitarios, para que estos puedan generar beneficios a corto y largo plazo para toda la comunidad y el entorno. Como último grupo, se encuentra el consejo universitario, su principal interés se basa en ejecutar un plan de mejorar las políticas y actividades ambientales las cuales se llevan a cabo de dentro de la universidad.

Para finalizar, se indaga acerca de los problemas percibidos de cado uno de los grupos previamente estructurados, en este caso nos centramos específicamente en el grupo estudiantil, el cual es importante resaltar que deben existir políticas de cuidado del medio ambiente, para incentivar a trabajar en un plan concreto de manejo de residuos que los ayude a comprender la importancia de respetar y cuidar los recursos naturales. Y empezar a inculcar a motivar a los estudiantes hacer campañas de reciclaje, para generar el buen manejo de los residuos, evitando de esta manera la contaminación del medio ambiente.

\section{Conclusiones}

En los últimos años se ha podido evidenciar un creciente interés ambiental por parte de la sociedad con un enfoque hacia la sostenibilidad. Esto quiere decir, que la mayoría de las personas han tomado en consideración los efectos que han sido generados a partir del calentamiento global, insertándose a la búsqueda de un equilibrio orientado a fines sostenibles. De igual manera, se da a conocer un hito transcendental que ha marcado las diversas normativas y leyes de varios países que tienen como propósito incentivar a las personas hacia la cultura ambiental, el denominado Protocolo de Kioto originado en Japón en 1997 aunque en el año 2005 entro en vigor. El cual, tiene como fin reducir los gases de efecto invernadero, ya que son los principales causantes del calentamiento global. Además, de que promueve un desarrollo sustentable para los países que se encuentran involucrados en el protocolo conocidos como países en desarrollo.

Cabe resaltar que dentro del protocolo el principio central del mismo hace referencia a presentar una responsabilidad común, pero a su vez diferenciada. Tomando en consideración, que los países deben comprometerse a presentar actividades que generen una responsabilidad enfocada en el medioambiente, aunque para cada país será totalmente diferente de acuerdo a sus necesidades. En el caso, de la Agenda 2030 para el Desarrollo Sostenible, plantea un total de 17 objetivos y 169 metas que reflejan una dinámica integrada con respecto a aspectos económicos, sociales y ambientales. A partir, de la Agenda 2030 surge los 17 objetivos o también conocidos como los ODS, los cuales se encuentran adoptados de manera global y pretenden poder erradicar con la 
pobreza, el proteger al planeta y afirmar la prosperidad para los demás. Es por eso, que cada objetivo presenta una meta de carácter específico. Dentro de los objetivos que se relacionan con aspectos ambientales son el objetivo 12, el cual manifiesta una producción y consumo responsable, el objetivo 13 la acción por el clima, el objetivo 14 la vida submarina y el objetivo 15 la protección de vida en ecosistemas terrestres.

La Universidad y el medio ambiente se encuentran relacionados entre sí, por el mismo hecho de que las universidades pretenden diseñar actividades y acciones que posibiliten el desarrollo de habilidad y capacidades para la conservación del Medio Ambiente. Cabe mencionar, que el medioambiente es una de las preocupaciones tanto políticas, económicas, sociales y educativas a nivel mundial. Por tal motivo, las instituciones de carácter educativos destacan la importancia del conocimiento y el cumplimiento de las normas ambientales que se encuentran vigentes en los países. A su vez, de repercutir en una formación ambiental de los jóvenes universitarios creando un comportamiento social responsable antes la problemática ambiental evidenciada, es decir, fomentar a la creación de una cultura ambiental desde la formación académica.

La Universidad Metropolita o también denominada UMET fue creada en el año 2000 y presenta su matriz en la ciudad de Guayaquil y sus sedes en las ciudades de Quito y Machala. A lo largo de su trayectoria como ente educativo ha realizado diversos proyectos de investigación y vinculación con la sociedad los cuales presentan un enfoque de sostenibilidad. Cabe recalcar que esta publicación se presenta como resultado del proyecto de investigación titulado "Gestión de empresas y grupos de interés hacia la sostenibilidad desde la Responsabilidad Social Empresarial" implementado en la Universidad Metropolitana sede Machala, mismo que se encuentra conformado por 10 docentes y 14 estudiantes de la carrera Administración de Empresas.

Dentro de la presente investigación se planteó la incógnita de “¿Cómo percibe la Comunidad Universitaria las acciones ambientales en la UMET Sede Machala?" De igual forma se estipularon los objetivos específicos para en si recabar información, los cuales fueron; Evaluar las acciones ambientales, determinar el conocimiento ambiental de la comunidad universitaria, establecer el nivel de compromiso de la comunidad universitaria para la implementación de acciones ambientales, identificar las acciones ambientales a implementar por parte de la UMET.

Por parte de las acciones ambientales ejecutadas por la Universidad Metropolitana Sede Machala, se encuentran ya implementadas series de actividades las cuales permitan un mejor manejo de recursos y en si al cuidado del medio ambientales dentro de nuestro entorno, las acciones implementadas por la UMET son; crear conciencia sobre el uso de los recursos no renovables, campañas de reciclaje, capacitaciones en educación ambientales, crear y mantener espacios verdes dentro y fuera de las instalaciones y limpieza de entrono naturales.

Es pertinente mencionar que esta investigación surge como resultado del proyecto de investigación titulado Gestión de empresas y grupos de interés hacia la sostenibilidad desde la Responsabilidad Social Empresarial, utilizando como objeto de estudio a la comunidad universitaria de la UMET sede Machala, con la finalidad de conocer la percepción frente a las acciones proambientales implementadas y verificar la predisposición e interés para participar activamente en futuras actividades que la universidad ejecute en beneficio y cuidado del 
medioambiente. Cabe recalcar que dentro de las principales limitaciones del estudio se considera que la pandemia por Covid-19 ha constituido un factor relevante para que los miembros de la comunidad universitaria no hayan participado activamente en actividades proambientales generadas por la Universidad Metropolitana, debido a la virtualidad con la que se manejan los procesos académicos y de gestión. Por tal razón es recomendable que se replique la investigación cuando se retorne a la presencialidad para contrastar los resultados y tomar las medidas respectivas.

Para finalizar, se puede concluir que la Universidad Metropolitana Sede Machala se encuentra completamente comprometida con la sociedad y con el medio ambiente, de igual forma busca crear conciencia ambiental dentro de la comunidad universitaria, para que así obtengan un mejor uso de los recursos y del cuidado ambiental para reducir el impacto generado por la deficiente educación ambiental. Además, los congresos y actividades con enfoque de sostenibilidad ambiental que ha implementado la UMET sede Machala constituyen factores relevantes dentro de la comunidad universitaria, ya que obtienen una gran acogida y demuestra el alto compromiso con las acciones proambientales que se ejecutan dentro y fuera de la institución educativa.

\section{Referencias bibliográficas}

Aparicio, X., Chininin, M., y Toledo Rodríguez, O. (2017). El Rol de la Vinculación en la integración de las funciones sustantivas de la Universidad Metropolitana del Ecuador. $\begin{array}{llll}\text { Revista Universidad } & y & \text { Sociedad, } & 9(4)\end{array}$ http://scielo.sld.cu/scielo.php?script=sci_arttext\&pid=S2218-36202017000400005

Bolaños Sánchez, V. H., Ortega Garnelo, F., y Reyes Baza, D. (2015). Medio ambiente, ciencia y sociedad. Andamios, 12(29) 7-14. http://www.scielo.org.mx/scielo.php?script=sci_arttext\&pid=S1870-00632015000300007

Bybee, R. (1991). Planet earth in crisis: how should science educators respond. The American Biology Teacher, 53(3), 146 - 153.

CACES. (2021). Quienes somos. Consejo de Aseguramiento de la Calidad de la Educación Superior. https://www.caces.gob.ec/quienes-somos/

Cancino, J., y Morales, M. (2008). Responsabilidad social empresarial. Universidad de Chile. http://repositorio.uchile.cl/bitstream/handle/2250/122747/Cancino_Morales_2008.pdf;

CEPAL. (2016). Agenda 2030 y los Objetivos de Desarrollo Sostenible. Una oportunidad para América Latina y el Caribe. Comisión Económica para América Latina y el Caribe. https://bit.ly/3r8qC6g

Cuéllar Caicedo, F., y Méndez Céspedes, P. (2006). Concepciones sobre Educación Ambiental de Docentes de Programas de Licenciatura en Educación Ambiental o Afines. Hallazgos, (6), 183-204. https://www.redalyc.org/articulo.oa?id=413835165012

Escobar Escobar, M., y Escobar, R. (2018). Construcción y aplicación de un árbol de problemas transdisciplinar en práctica asistencial. Ciencia y Salud Virtual, 10(2) 14-31. https://dialnet.unirioja.es/servlet/articulo?codigo $=6732637$

Gallopín, G. C. (2003). Sostenibilidad y desarrollo sostenible: un enfoque sistémico. CEPAL. https://bit.ly/317CTE6 
Ghitis, T., y Alba, A. (2019). Percepciones de futuros docentes sobre el uso de tecnología en educación inicial. Revista electrónica de investigación educativa, 2-5. https://doi.org/10.24320/redie.2019.21.e23.2034

Gómez Gil, C. (2018). Objetivos de Desarrollo Sostenible (ODS): una revisión critica. Papeles de relaciones eco sociales y cambio global, (140), 107-118. https://dialnet.unirioja.es/servlet/articulo?codigo=6312616

Hernández Sampieri, R., y Mendoza Torres, C. P. (2018). Metodología de la investigación: Las rutas cuantitativa, cualitativa y mixta. México: McGraw Hill.

Hernández-Hernández, N., y Garnica-González, J. (2015). Árbol de Problemas del Análisis al Diseño y Desarrollo de Productos. Conciencia Tecnológica, (50), 38-46. https://www.redalyc.org/pdf/944/94443423006.pdf

Jurado, A. (2021). Economía circular: conversión de residuos en recursos, de Xavier Elías Castells. Ambiente En Diálogo, en (2), http://ojs.opds.gba.gov.ar/index.php/aed/article/view/18

Laportilla Estévez, N. D., García Ferrandis, I., Santos Abreu, I. C., Fragoso Martínez, A. J., y Aguilar Guillermo, E. (2020). Dimensión ambiental en la formación del profesorado en el marco de los objetivos de desarrollo sostenible. Indagatio Didáctica, 12(4), 363-382. https://doi.org/10.34624/id.v12i4.21802

Luis García, E. (2018). El medio ambiente sano: La consolidación de un derecho. Iuris Tantum Revista Boliviana de Derecho, 550-559. http://www.scielo.org.bo/scielo.php?script=sci_arttext\&pid=S2070-

$81572018000100019 \& \operatorname{lng}=\mathrm{es} \&$ tlng=es

Maldonado-Villalpando, E., Herrera Torres, H. A., y García Rojas, H. R. G (2018). Diseño de política ambiental e innovación social. Aportaciones teórico-metodológicas para la gestión sustentable de áreas naturales protegidas. Economía y Sociedad,22(38), 111-128. https://www.redalyc.org/journal/510/51058253008/html/

Morocho, F. R. A. (2018). La economía circular como factor de desarrollo sustentable del sector productivo. INNOVA Research Journal, 3(12), 78-98. https://doi.org/10.33890/innova.v3.n12.2018.786

Mckeown, R. H. (2002). Manual de Educación para el Desarrollo Sostenible. Waste Management Research and Education Institute. https://bit.ly/3cHmtO8

Niño Benavides, T. D. P., y Cortés, M. I. (2018). Comunicación estratégica y responsabilidad social empresarial, escenarios y potencialidades en creación de capital social: una revisión de la literatura. Revista Prisma Social, (22), 127-158. https://revistaprismasocial.es/article/view/2570

ONU. (1987). Informe Bruntland, Our commun future. Naciones Unidas. https://sustainabledevelopment.un.org/content/documents/5987our-common-future.pdf

Pardavé, W. (2007). Estrategias ambientales de las 3R a las 10R. ECOE Ediciones.

Pérez, M. M., Maldonado, I. P., y Uzcátegui, S. B. (2007). El marco lógico y las organizaciones educativas. Contribución metodológica para la mejora de la escuela. Sapiens. Revista Universitaria de Investigación, 8(2), 147-167. https://www.redalyc.org/articulo.oa?id=41080210

Posada González, N. L. (2017). Algunas nociones y aplicaciones de la investigación documental denominada estado del arte. Investigación Bibliotecológica, 31(73), 237-263. https://doi.org/10.22201/iibi.24488321xe.2017.73.57855. 
Reyes de Blobfeld, E. (2014). La responsabilidad social empresarial y la justicia restaurativa como factores clave del Proyecto Alcatraz de la Fundación Santa Teresa. Sapienza Organizacional, 1(1), 21-44. https://www.redalyc.org/pdf/5530/553056603004.pdf

Sarmiento, S. (2010). Gestión estratégica: clave para la responsabilidad social de las empresas. Dimensión https://www.researchgate.net/publication/277265517_Gestion_estrategica_clave_para_la _responsabilidad_social_de_las_empresas

UMET. (2019). Ejes temáticos. IV Congreso Internacional Medio Ambiente y Desarrollo Sostenible: https://4tocongreso.umetmachala.edu.ec/ejes-tematicos/

Valera Mejía, F., y Silva Naranjo, E. (2012). Guía de capacitación en educación ambiental y cambio climático. Guía de capacitación en educación ambiental y cambio climático. The Nature Conservancy. https://bit.ly/3cO9n1w

Vargas, C., Rosario, R., y Briones, C. D. (2017). Impacto de la materia desarrollo sustentable en el cambio de la conciencia ambiental de los estudiantes. Luna Azul (45), 3-10. https://doi.org/10.17151/luaz.2017.45.2 